\title{
Nearly Automated Left Ventricular Long Axis Tracking on Real Time Three-Dimensional Echocardiographic Data
}

\author{
F Veronesi ${ }^{1,2}$, C Corsi $^{1}$, EG Caiani ${ }^{2}$, C Lamberti $^{1}$ \\ ${ }^{1}$ Dipartimento di Elettronica, Informatica e Sistemistica, Università di Bologna, Italy \\ ${ }^{2}$ Dipartimento di Bioingegneria, Politecnico di Milano, Italy
}

\begin{abstract}
The measurement of left ventricular (LV) long axis length (LAL) is an integral part of echocardiographic evaluation of $L V$ volume, the most important determinant of $L V$ systolic function. LAL measurements from $2 D$ echocardiography (2DE) are highly dependent on the ability to obtain non-foreshortened LV images. Real-time $3 D$ echocardiography (RT3DE) could potentially overcome the effects of long-axis foreshortening, but LAL measurements on RT3DE data are currently based on manual analysis and are time-consuming. We developed and tested a nearly automated method based on optical flow techniques for the measurement of the LV LAL throughout the cardiac cycle from RT3DE data. Results of comparisons on 10 patients with manual tracing on RT3DE data showed good agreement and no significant bias $(r=.99$, bias $=-1.8 \mathrm{~mm})$. The proposed method allowed fast and accurate quantification of the LAL throughout the cardiac cycle with minimal user interaction and short computational time.
\end{abstract}

\section{Introduction}

Longitudinal LV shortening defined as the difference between the long-axis length (LAL) at end diastole (ED) and at any other instant in the cardiac cycle, is an important parameter in the evaluation of left ventricular (LV) systolic function [1-2]. Moreover, an incorrect estimate of LAL could affect the measurements of crucial clinical parameters, such as LV volume, which is obtained from these estimates using geometric modelling. However, conventional two-dimensional echocardiographic (2DE) measurements of the LAL are subjective and time-consuming and rely on the ability to obtain nonforeshortened apical long axis views [3].

Real-time 3D echocardiography (RT3DE) is an emerging imaging technique that allows fast acquisition of volumetric datasets from a single transthoracic acoustic window. This new technology provides more complete information on left ventricular (LV) anatomy and function, which has recently triggered research protocols aimed at its testing and validation. By using advanced image processing techniques, it is possible to obtain from RT3DE data not only qualitative 3D visual information on chamber shape, function and wall motion [4], but also quantitative clinically useful parameters of LV function [5].

A recent paper [6] has highlighted the importance of selecting anatomically correct, non-foreshortened apical cross-sections from the RT3DE data for accurate estimates of LV mass. The major cause of the known underestimation of LV mass by 2DE was shown to be apical foreshortening [6]. However, in this study, the selection of the non-foreshortened apical views was performed off-line by manual analysis of RT3DE data. Because of the time consuming nature of this procedure, it was limited to end-diastolic (ED) and end-systolic (ES) frames and thus did not provide information on LAL changes throughout the cardiac cycle.

We hypothesized that LV long axis could be automatically identified throughout the cardiac cycle from the RT3DE, and thus could provide the basis for fast, automated quantification of LV longitudinal shortening. Accordingly, the aim of this study was to develop a nearly automated method, based on optical flow techniques [7], to detect frame-by-frame the LV long-axis and measure its length. The automated LAL measurements were validated by comparisons with manual frame-by-frame measurements performed on the same RT3DE datasets.

\section{Methods}

\subsection{Data acquisition}

Ten patients (age $52 \pm 12$ years) with transthoracic acoustic windows that allowed adequate endocardial visualization without contrast enhancement were studied. RT3DE imaging was performed using a commercial ultrasound scanner (SONOS 7500, Philips) equipped with a fully sampled matrix array transducer $(\mathrm{X} 4,2-4 \mathrm{MHz})$ operating in the harmonic mode. RT3DE datasets were acquired using the wide-angled mode, wherein four wedge-shaped sub-volumes $\left(93^{\circ} \times 21^{\circ}\right)$ were acquired over 
four cardiac cycles during a breath-hold with ECG gating. Care was taken to include the entire LV cavity within the pyramidal 3D scan volume.

\subsection{Study design}

In each patient, the RT3DE data were analyzed using two methods described below: (1) manual frame-byframe measurement of LAL; (2) automated tracking of LV long-axis throughout the cardiac cycle using the optical flow technique, followed by computation of LAL. These manual and automated LAL measurements were compared using linear regression and Bland-Altman analyses. In addition, percent discordance was calculated as the sum of frame-by-frame inter-technique differences between the measured LAL values.

\subsection{Long axis selection procedure}

In order to assess the LV long axis position throughout the cardiac cycle, the coordinates of the points representing the center of the mitral annulus and the apex of the LV chamber were manually selected on the first frame only. To this effect, twelve cross-sectional long axis views $\left(15^{\circ}\right.$ apart) were displayed to allow the operator first to select the projection in which the mitral annulus was best visualized. Then, on that plane the operator manually selected the two points of insertion of the mitral leaflets (Figure 1, left, points A and B). On a second plane, perpendicular to the first one and passing through the center $\mathrm{M}$ of the line connecting points $\mathrm{A}$ and B, two additional points were selected (Figure 1, right, points $\mathrm{C}$ and D). Finally, the center of the mitral annulus was calculated as the center of the line connecting points $\mathrm{C}$ and $\mathrm{D}$ (point $\mathrm{X}$ ). From the volumetric display, the LV apical tip was then selected. The straight line connecting
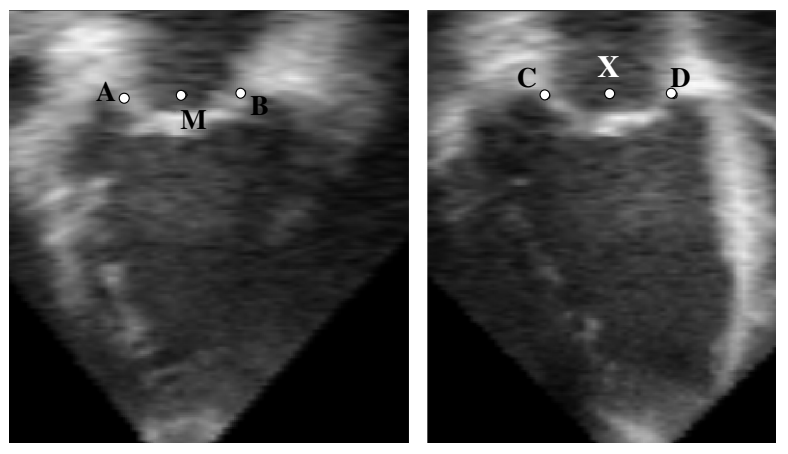

Figure 1. Detection of the center of the mitral valve: on the selected cut-plane (left), two points, A and B, were selected at the insertion of the anterior and posterior mitral leaflets. On the orthogonal plane (right) passing through the center $\mathrm{M}$ of the segment $\underline{A} \underline{B}$, two additional mitral annulus points, $\mathrm{C}$ and $\mathrm{D}$, were selected. The center of the mitral annulus $\mathrm{X}$ was then computed as the mid-point of the segment $\underline{\mathrm{CD}}$. point $\mathrm{X}$ with the apex represented the initial long axis position for the optical flow algorithm, and its length was computed as the Euclidean distance between these two points. To obtain the manual reference LAL values, the same point selection procedure was applied frame-byframe throughout the cardiac cycle.

\subsection{Optical flow}

The tracking of the three initialized points $(C, D$ and the apex) defining the LV long axis position was performed using a two-step optical flow technique. First, the Lucas-Kanade optical flow algorithm [7] was applied. It is based on the assumption that the brightness $I(\mathbf{x}, t)$ in the image is conserved during motion:

$$
d I(\mathbf{x}, t) / d t=0 .
$$

In order to evaluate the velocity field $\mathbf{V}$ between two frames, a weighted least-square minimization of the following function is performed:

$$
\sum_{\mathbf{x} \in \Omega} W^{2}(\mathbf{x})\left[\nabla I(\mathbf{x}, t) \cdot \mathbf{v}+I_{t}(\mathbf{x}, t)\right]^{2}
$$

where the terms inside the square brackets are obtained by separating derivatives from (1), $\Omega$ is the domain containing the neighborhood of $\mathbf{x}$ (the initial point to be tracked) and $W(\mathbf{x})$ is a window, that gives more importance to central terms rather than for the ones in the periphery. The solution of the minimization of (2) is obtained by isolating $\mathbf{V}$ in matrix form:

$$
\mathbf{v}=\left[A^{T} W^{2} A\right]^{-1} A^{T} W^{2} \mathbf{b}
$$

where

$$
\begin{aligned}
& A=\left[\nabla I\left(\mathbf{x}_{1}\right), \ldots, \nabla I\left(\mathbf{x}_{\lambda}\right)\right]^{T} \\
& W=\operatorname{diag}\left[W\left(\mathbf{x}_{1}\right), \ldots, W\left(\mathbf{x}_{\lambda}\right)\right], \\
& \mathbf{b}=-\left(I_{t}\left(\mathbf{x}_{1}\right), \ldots, I_{t}\left(\mathbf{x}_{\lambda}\right)\right)^{T} .
\end{aligned}
$$

Once the velocity is known, the displacement of the point of interest is calculated as $\mathbf{d}_{L K}=\mathbf{v} \Delta t$, and the position of the point $\mathbf{x}$ in the next frame is given by :

$$
\mathbf{x}_{L K}=\mathbf{x}+\left[\mathbf{d}_{L K}\right],
$$

where square brackets mean rounding approximation.

As a second step, a block matching algorithm was applied, in order to evaluate the exact position of $\mathbf{x}$ into the next frame $\left(\mathbf{x}_{t+1}\right)$. For each point inside a new 3D window $\mathbf{V}\left(\mathbf{x}_{L K}\right)$ centered in $\mathbf{x}_{L K}$, another $3 \mathrm{D}$ window $\mathbf{F}$ (x) was defined and compared to the window of the same size around $\mathbf{x}$, by computing the sum of squared differences $\phi\left(\mathbf{x}_{t+1}\right)$ : 


$$
\phi\left(\mathbf{x}_{t+1}\right)=\sum_{i=-m}^{m} \sum_{j=-m}^{m} \sum_{k=-m}^{m} F(i, j, k)\left[\begin{array}{l}
I_{t}(\mathbf{x}+(i, j, k))+ \\
-I_{t+1}\left(\mathbf{x}_{t+1}+(i, j, k)\right)
\end{array}\right]^{2}
$$

The point corresponding to the minimum of $\phi\left(\mathbf{x}_{t+1}\right)$ is the one that best fits the window around the starting point. The final point was then computed as:

$$
\mathbf{x}_{t+1}^{\text {final }}=\arg \min \phi\left(\mathbf{x}_{L K}^{i}\right) \quad \text { for } \mathbf{x}_{L K}^{i} \in V\left(\mathbf{x}_{L K}\right)
$$

Therefore the final point was evaluated as the sum of the displacements computed from the two steps of tracking:

$$
\mathbf{x}_{t+1}^{\text {final }}=\mathbf{x}_{L K}+\mathbf{d}_{M}=\mathbf{x}_{t}+\left[\mathbf{d}_{L K}\right]+\mathbf{d}_{M},
$$

with $\mathbf{x}_{t}$ as the initial point at time $\mathrm{t},\left[\mathbf{d}_{L K}\right]$ as the rounded displacement evaluated by the Lucas-Kanade algorithm, and $\mathbf{d}_{M}$ as the displacement evaluated by the block matching technique.

The resulting point $\mathrm{X}_{t+1}^{\text {final }}$ was considered as the starting point for the tracking on the following frame. In this way the points selected by the operator were followed frame by frame throughout the cardiac cycle and the LAL was computed as the Euclidean distance between mitral valve center and apex.

\section{Results}

For each subject, the RT3DE analysis with the proposed nearly automated technique, including data retrieval, selection of points, automated computation of the center of the mitral valve and dynamic tracking of the LV long axis throughout the cardiac cycle, required between 1 and 2 minutes, using a personal computer (Pentium IV, 2.6GHz, 1Gb RAM). The frame-by-frame manual analysis of one complete cardiac cycle required between 4 and 6 minutes. An example of the rendered volume in different $3 \mathrm{D}$ perspectives with the initialized
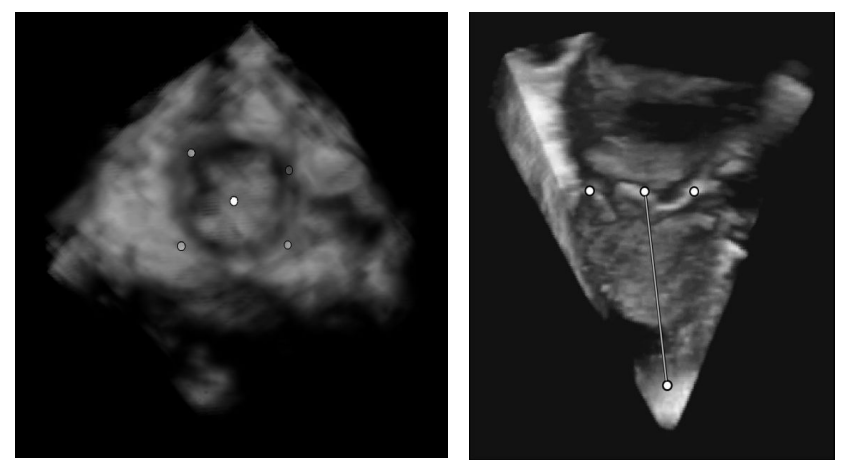

Figure 2. Left: example of a rendered volume with the four points selected by the operator and the automatically determined center of the mitral valve. Right: superimposition to a rendered RT3DE cross-section of the long axis.

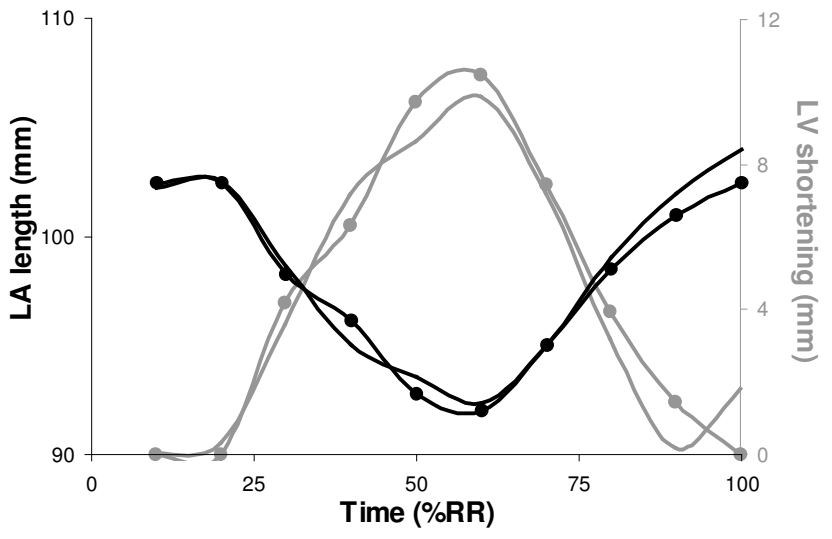

Figure 3. Example of the LAL (black lines) over time in percentage of the heart cycle duration (\%RR); curves obtained from a RT3DE dataset by automated analysis (with symbols) and by manual tracing (without symbols), together with the corresponding LV shortening (secondary y-axis, gray lines, with and without symbols respectively).

points and long axis superimposed is shown in figure 2 .

The manually measured LAL at ED ranged between subjects from $70.3 \mathrm{~mm}$ to $100.3 \mathrm{~mm}$. The LV shortening was $15 \pm 12 \%$ of the ED length.

Figure 3 shows an example of the LAL measured over time (in \% of the RR interval) and the derived LV shortening, resulting from both the automated algorithm and the manual analysis. The close correspondence between the curves in this example reflect excellent intertechnique agreement in every frame throughout the cardiac cycle.

Linear regression analysis between LAL obtained frame-by-frame in the 10 patients with the two techniques showed an excellent correlation $(\mathrm{r}=0.99)$ with a regression line of $\mathrm{y}=0.94 \mathrm{x}+5.3$ (Figure 4, top). The standard error of the estimate was $1.8 \%$. Bland-Altman analysis showed no significant bias $(-0.18 \mathrm{~mm}$, corresponding to $-0.2 \%$ of the mean value) versus manual measurements, with narrow $95 \%$ limits of agreement $(3.82 \mathrm{~mm})$ (Figure 4, bottom). The calculated percentage of discordance was $1.73 \% \pm 0.57 \%$ (range: $0.60 \%$ $2.38 \%$ ), confirming the excellent rate of agreement between the two techniques. Moreover, the paired t-test evidenced no significant differences between the two groups of measurements.

\section{Discussion and conclusions}

RT3DE provides volumetric data that allows avoiding the foreshortening problem that has been affecting 2DEderived LV long-axis measurement, and thus promises to allow more accurate assessment of clinical parameters related to LV function [1-4]. However, no semiautomated or automated tool is currently available to 

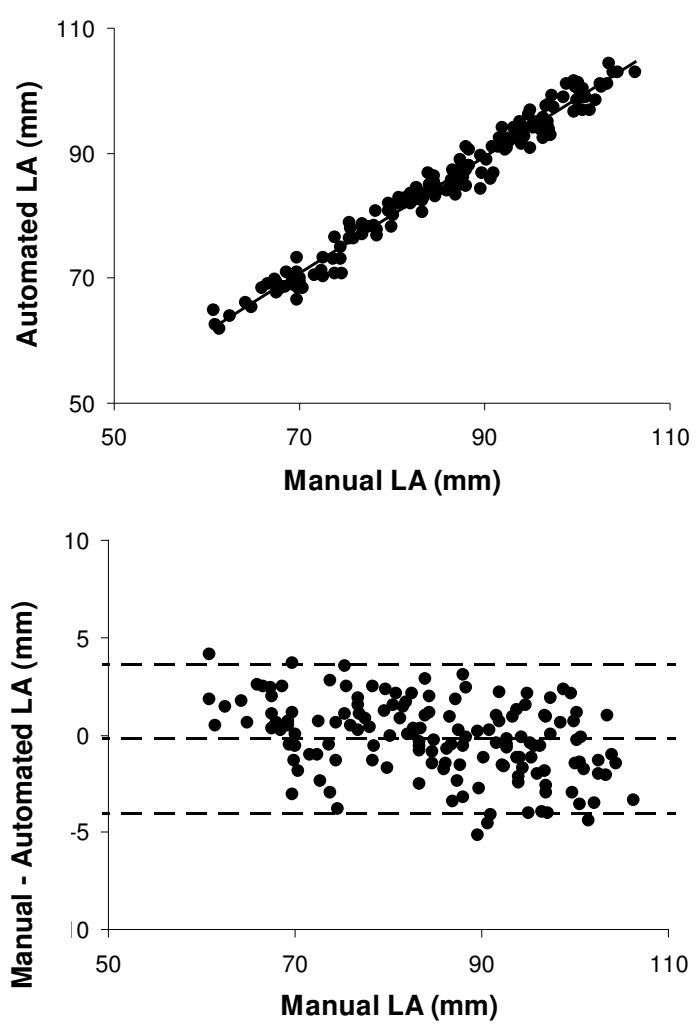

Figure 4. Results of the linear regression (top) and Bland Altman (bottom) analyses of the frame-by-frame measurements of the LV LA obtained by RT3DE manual tracing versus the proposed automated procedure in the analyzed population $(\mathrm{N}=10)$

track and measure the LAL on RT3DE data. To avoid the time consuming procedure of manual identification and measurement of the LV long axis throughout the cardiac cycle, we developed a fast method that requires minimal user interaction for the automated frame-by-frame tracking of the LV long axis and for the computation of its length. The proposed method employs in cascade two optical flow techniques, in order to minimize the computation time and the effects of noise. This technique was tested in a small group of subjects and results compared with manually derived LAL values showed excellent agreement.

In conclusion, the proposed method for LV long axis tracking and LV longitudinal shortening measurements is fast and reliable, and could be used to assess LV function from RT3DE datasets. Moreover, it may prove useful in automated identification of non-foreshortened apical views and thus allow improved evaluation of $\mathrm{LV}$ volumes, which is of crucial importance in multiple clinical scenarios.

\section{Acknowledgements}

The authors thank Roberto Lang, MD, Victor MorAvi, PhD and Lynn Weinert, BS, of the Noninvasive Cardiac Imaging Laboratories, University of Chicago Medical Center, for their invaluable contribution.

\section{References}

[1] Alam M, Hoglund C, Thorstrand C. Longitudinal systolic shortening of the left ventricle: an echocardiographic study in subjects with and without preserved global function. Clin Physiol 1992;12(4):443-452.

[2] Ozer N, Can I, Atalar E, Sade E, Aksoyek S, Ovunc K, Aytemir K, Tokgozoglu L, Ozmen F, Kes S. Left ventricular long-axis function is reduced in patients with rheumatic mitral stenosis. Echocardiography 2004;21(2):107-112.

[3] Schiller NB, Shah PM, Crawford M, DeMaria A, Devereux $\mathrm{R}$, Feigenbaum H, Gutgesell H, Reichek N, Sahn D, Schnittger I. Recommendations for quantitation of the left ventricle by two-dimensional echocardiography. American society of echocardiography committee on standards, subcommittee on quantitation of two-dimensional echocardiograms. J Am Soc Echocardiogr 1989;2(5):358367.

[4] Sugeng L, Weinert L, Lang RM. Left ventricular assessment using real time three dimensional echocardiography. Heart 2003;89(Suppl 3):29-36.

[5] Caiani EG, Corsi C, Zamorano J, Sugeng L, Maceneaney P, Weinert L, Battani R, Gutierrez JL, Koch R, Perez de Isla L, Mor-Avi V, Lang RM. Improved semiautomated quantification of left ventricular volumes and ejection fraction using 3-dimensional echocardiography with a full matrix-array transducer: comparison with magnetic resonance imaging. $\mathrm{J}$ Am Soc Echocardiogr 2005; 18(8):779-88.

[6] Mor-Avi V, Sugeng L, Weinert L, MacEneaney P, Caiani EG, Koch R, Salgo IS, Lang RM. Fast measurement of left ventricular mass with real-time three-dimensional echocardiography: comparison with magnetic resonance imaging. Circulation 2004;110(13):1814-1818.

[7] Barron JL, Fleet DJ, Beauchemin SS. Performance of optical flow techniques. International Journal of Computer Vision, 1994:12(1):43-77.

Federico Veronesi

Laboratorio di Bioingegneria

Via Risorgimento 2,

40100 Bologna, Italy

fveronesi@deis.unibo.it 